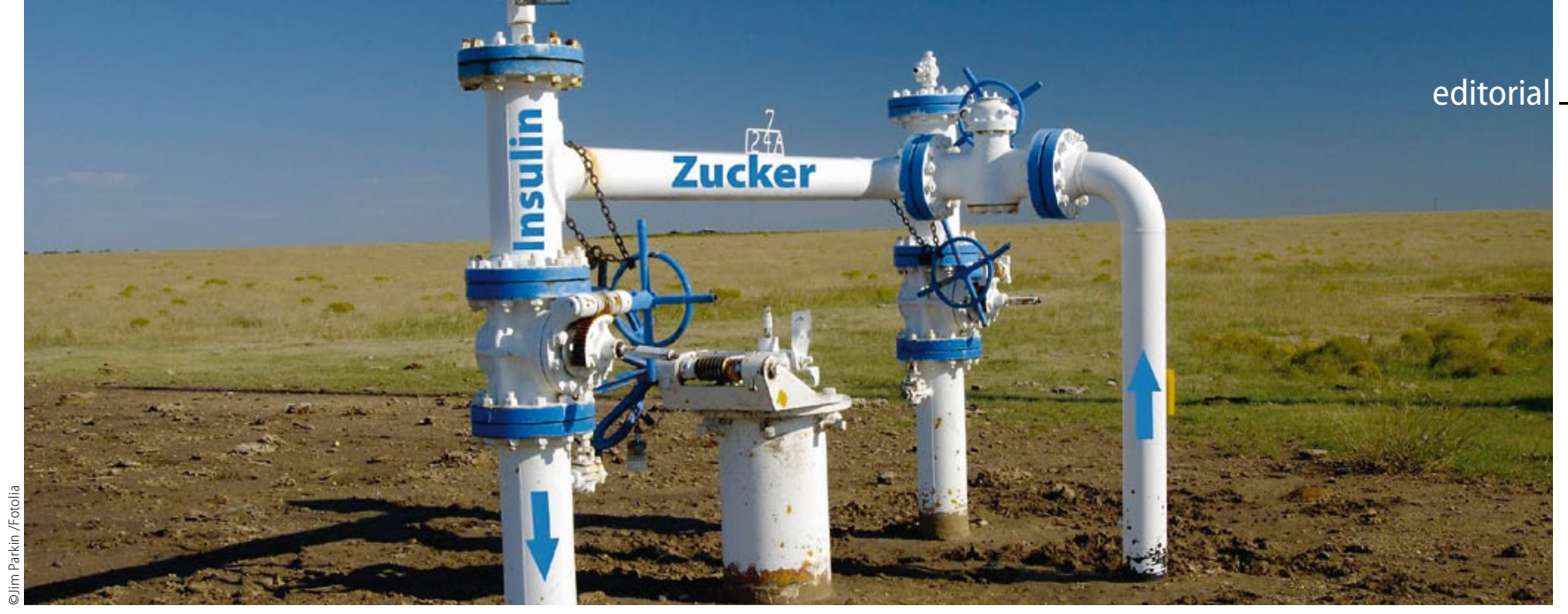

Die Henne-oder-Ei-Frage beim Typ-2-Diabetes

\title{
Was ist zuerst da - Insulinresistenz oder Hyperinsulinämie?
}

Bei dem bisherigen pathophysiologischen Modell der Pathogenese des Typ-2-Diabetes geht man von einer Insulinresistenz als Basis aus. Die fehlende Wirksamkeit versucht der Organismus durch eine Mehrproduktion von Insulin zu kompensieren. Wenn die Hyperinsulinämie nicht mehr ausreicht, kommt es zu einem Anstieg der Blutglukose. So weit, so gut.

Aber: In der Dezemberausgabe 2012 von Diabetes Care wird dieses Modell in zwei unabhängigen Beiträgen in Frage gestellt. Walter Pories und Lynis Dohm (Brody School of Medicine, East Carolina University, Greenville, North Carolina, USA) weisen in ihrer Argumentation darauf hin, dass es bei der Pathogenese des Typ-2-Diabetes zu einem kontinuierlichen Anstieg der basalen Insulinproduktion kommt, die auch noch lange nach Manifestation der Erkrankung nachzuweisen ist [1]. Im Vergleich zu gesunden schlanken Kontrollpersonen konnten sie in eigenen Studien bei übergewichtigen Personen mit Typ-2-Diabetes und Nüchternglukosewerten über $140 \mathrm{mg} / \mathrm{dl}$ bis zu 9-fach erhöhte basale Insulinspiegel nachweisen. Interessanterweise sinken die basalen Insulinspiegel schon innerhalb der ersten Woche nach einer bariatrischen Operation mit Bildung eines Rouxen-Y-Magenbypasses ab. Die bei Typ-2-Diabetes fehlende bzw. deutlich reduzierte postprandiale Insulinsekretion, die bei dem bisherigen pathogenetischen Modell im Vordergrund steht, lässt sich nach einer solchen Operation auch wieder nachweisen.

Nach der Ansicht der Autoren wird durch die Magenbypassoperation ein diabetogenes Signal aus dem Darm ausgeschaltet, das die Hyperinsulinämie verursacht.

Barbara Corkey vom Obesity Research Center, University School of Medicine in Boston, USA argumentiert in ihrem Beitrag ähnlich. Sie postuliert, dass die basale Insulinsekretion durch Monoglyzeride, künstliche Süßstoffe und Eisen erhöht wird [2]. Dabei scheinen Sauerstoffradikale, Stickstoffmonoxid und Peroxide beteiligt zu sein. Durch Hyperinsulinämie ohne entsprechende Nährstoffe kommt es zu einem Abfall der Blutglukose und vermehrte Nahrungsaufnahme. Bedingt durch die Hyperinsulinämie werden periphere Insulinrezeptoren herunter geregelt und es entsteht erst dann eine Insulinresistenz. Die Autoren folgern, dass wenn ihre Hypothese korrekt wäre, müss- te die frühe Insulintherapie bei Typ-2-Diabetes überdacht werden. Denn man behandelt Hyperthyreose ja auch nicht mit Schilddrüsenhormon und M. Cushing nicht mit Kortison [1]. Die Argumentation ist spannend und könnte den mäßigen Erfolg in der langfristigen Therapie bei Typ-2-Diabetes erklären.

\section{Stimulierung der Insulinproduktion wäre kontraindiziert} Was wären die Konsequenzen, wenn sich diese Hypothese als wahr herausstellen würde? Primäres Ziel der Typ-2-DiabetesTherapie wäre es dann, bei den Betroffenen jene Faktoren zu eliminieren, welche die Insulinproduktion stimulieren. Sämtliche Medikamente, die die Insulinspiegel steigern (Sulfonylharnstoffe, Glinide oder Insulin) wären dann kontraindiziert. Stattdessen würden kohlenhydratarme Fastenkuren eingesetzt.

Die Forschung müsste dann auch mehr versuchen, die Interaktionen zwischen Umwelt und Verhalten zu verstehen, die die Hyperinsulinämie fördern. Die Inhalte von Ernährungsberatungen würden sich deutlich ändern, und die bisherige Empfehlung, Fett zu meiden, würde - zumindest im Hinblick auf die Manifestation eines Diabetes mellitus - ebenfalls verändert. Denn dann würde die Zuckerkrankheit ja durch den Zucker ausgelöst....

1. Pories WJ, Dohm GL. Diabetes Care 2012;35: 2438-42

2. Corkey BE. Diabetes Care. 2012;35:2432-7

p.s. Übrigens gibt es ein interessantes neues Projekt auf Seite $9 \mathrm{im} \mathrm{Heft.}$

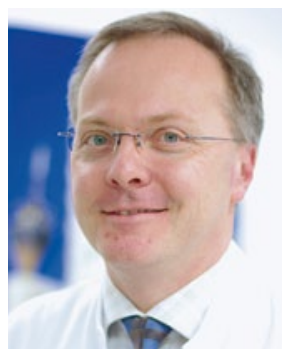

Professor Dr. med. Stephan Martin

Verbund der Katholischen Kliniken

Düsseldorf (VKKD)

Chefarzt für Diabetologie und Direktor

des Westdeutschen Diabetes- und

Gesundheitszentrum (WDGZ)

Hohensandweg 37, 40591 Düsseldorf

Stephan.Martin@vkkd-kliniken.de 